\title{
Extraction of Anthocyanin from Purple Cabbage Based on Artificial Neural Network
}

\author{
Zhang Dongfeng, Li Pingfan ${ }^{\text {a,* }}$ \\ School of Food and Biotechnology, Guangdong Industry Technical College, Guangzhou, Guangdong, \\ 510300, China \\ a email: lipinf@163.com \\ *corresponding author
}

Keywords: Purple Cabbage, Pigment Extraction, Extraction Conditions, Antioxidant Activity

\begin{abstract}
With fresh purple cabbage as raw material and hydrochloric acid solution as extractant, the scavenging effect of purple cabbage on super oxide anion radical and hydroxyl radical of anthocyanin extract was screened by single factor and orthogonal test. Extract antioxidant activity. The results showed that $0.3 \mathrm{~mol} / \mathrm{l}$ purple cabbage anthocyanin hydrochloride was used as the extraction agent, and the extraction process was the best. Purple cabbage anthocyanin [anthocyanin purple cabbage anthocyanin: anthocyanin water purple cabbage anthocyanin $=1: 1$ purple cabbage anthocyanin $\mathrm{g} / \mathrm{ml}$ purple cabbage anthocyanin (purple cabbage Anthocyanin) purple cabbage anthocyanin]: anthocyanin added with hydrochloric acid purple cabbage anthocyanin $=1: 5$ purple cabbage anthocyanin (volume ratio) In hydrochloric acid solution, anthocyanin was extracted at $60{ }^{\circ} \mathrm{C}$ for 2 hours, the extraction rate was $1.67 \%$. To some extent, the anthocyanin concentration of purple cabbage is $0-12 \mathrm{Mg} / \mathrm{ml}$ purple cabbage anthocyanin, with the increase of purple cabbage anthocyanin concentration, the effect of superoxide anion removal, the maximum clearance rate of purple cabbage anthocyanin is $31.1 \%$, purple cabbage anthocyanin IC anthocyanin 50 purple cabbage anthocyanin purple cabbage anthocyanin $2.301 \mathrm{mg} / \mathrm{ml}$ purple cabbage anthocyanin; the content of purple cabbage anthocyanin is 0-0.6 in the range of purple cabbage $\mathrm{Mg} / \mathrm{ml}$ anthocyanin, the concentration of anthocyanin in purple cabbage increased, the removal effect of hydroxyl radicals increased, the maximum removal rate was $54.7 \%$ Schiff base of anthocyanin in purple cabbage, $54.7 \%$ Schiff base of anthocyanin in purple cabbage, 0.956 anthocyanin in purple cabbage, and $9 \mathrm{mg} / \mathrm{ml}$ anthocyanin in purple cabbage.
\end{abstract}

\section{Introduction}

The natural pigment has high safety, and the anthocyanin and natural pigment have certain health care effect on the prevention and health care of the anthocyanin. Purple cabbage anthocyanin [1-2] purple cabbage anthocyanin. Purple cabbage anthocyanin can strongly absorb ultraviolet rays, play the role of UV shielding in vivo, so that the normal differentiation of purple cabbage anthocyanin and other life processes can be carried out normally. At the same time, it can prevent coronary heart disease and myocardial defect. Therefore, the growth of cancer cells is a natural pigment, purple cabbage anthocyanin [3-4], which has good development and application potential [1]. At present, purple cabbage anthocyanin is the main product of purple cabbage anthocyanin, which has been put on the market. It is used to alleviate the eyestrain caused by computer anthocyanin in purple cabbage. Purple cabbage anthocyanin [5] purple cabbage anthocyanin. As a natural food colorant, purple cabbage anthocyanin is safe and nontoxic, and has a broad application prospect in the field of purple cabbage anthocyanin. Anthocyanins in Purple Cabbage [6-7]. Under the condition of $55{ }^{\circ} \mathrm{C}, 25 \%$ ethanol and $\mathrm{pH}=2$, the contents of anthocyanin, Albert anthocyanin [8] anthocyanin and anthocyanin of purple cabbage were studied. The microwave-assisted extraction of anthocyanin of purple cabbage was used to determine the absorption of purple cabbage pigment (100 purple cabbage pigment and 525 purple cabbage pigment) to purple cabbage pigment at $100 \mathrm{~W}$ and $600 \mathrm{~W}$ With the change of microwave power, the absorptivity of anthocyanin increased, and the power of 
anthocyanin was 300W[2]. The extraction time of anthocyanin from purple cabbage was 50 min with distilled water as extractant. The liquid-solid ratio of anthocyanin in purple cabbage was 40:1. ( $\mathrm{ml} / \mathrm{g}$ ), side temperature $40{ }^{\circ} \mathrm{C}$, ultrasonic assisted extraction of anthocyanin from purple cabbage is the best, the extraction power is $80 \%$. Xu Yamin et al. The total reduction amount of anthocyanin in Purple Cabbage [10] was determined by the common purple cabbage anthocyanin method and the Russian blue method. When the anthocyanin concentration of purple cabbage is $0-1 \mathrm{mg} / \mathrm{ml}$, the reduction ability of anthocyanin increases with the increase of concentration. The antioxidant activity and scavenging rate of the anthocyanin (11) and other anthocyanins of purple cabbage and DPPH of purple cabbage were 0.35 When the acid salt solid ratio of mol / L is $4: 1(\mathrm{ml} / \mathrm{g}$ ), anthocyanin is extracted from purple cabbage for 120 s with $900 \mathrm{~W}$. When anthocyanin is extracted from purple cabbage, the concentration of anthocyanin is $0.05 \mathrm{mg} / \mathrm{ml}$ and $0.15 \mathrm{mg} / \mathrm{ml}$. The DPPH free radical scavenging rate of anthocyanin increases with the increase of concentration[3]. The concentration of purple cabbage solution is $0.15 \mathrm{mg} / \mathrm{ml}$, with the highest scavenging rate. Anthocyanins were extracted from fresh purple cabbage with hydrochloric acid solution. The effects of extraction time, temperature and concentration on anthocyanin extraction were studied. The best extraction conditions were determined, and the antioxidant activity of anthocyanin was further determined, which laid a theoretical foundation for the full development and utilization of anthocyanin.

\section{Materials and Methods}

\subsection{Materials and Reagents}

Fresh purple cabbage: buy in vegetable wholesale market purple cabbage anthocyanin. Purple cabbage anthocyanin hydrochloric acid (analytical pure): sigma; tris (hydroxymethyl) aminomethane (Tris $\mathrm{HCl}$ ) buffer solution, o-purple cabbage anthocyanin pyrogallol solution, purple cabbage anthocyanin, salicylic acid ethanol solution, FeSO purple cabbage anthocyanin 4 purple cabbage anthocyanin solution, ascorbic acid purple cabbage anthocyanin solution are all analytical pure.

Table 1 Level of orthogonal test factors

\begin{tabular}{|c|c|c|c|}
\hline \multirow{2}{*}{ Level } & \multicolumn{3}{|c|}{ Factor } \\
\cline { 2 - 4 } & Hydrochloric acid concentration & Extraction temperature & Extraction time \\
\hline 1 & 0.25 & 40 & 1 \\
\hline 2 & 0.3 & 50 & 1.5 \\
\hline 3 & 0.35 & 60 & 2 \\
\hline
\end{tabular}

\subsection{Instruments and Equipment}

Jj-2b homogenizer: hws-24 electric constant temperature water bath of Jintan ronghua Instrument Manufacturing Co., Ltd.: blast drying oven of Shanghai Yiheng scientific instrument purple cabbage anthocyanin Co., Ltd.: Shanghai Fuma purple cabbage anthocyanin Experimental Equipment Co., Ltd.; yp1002n electronic balance: Shanghai Precision Science wfj7200 visible spectrophotometer: Shanghai purple cabbage anthocyanin Instrument Co., Ltd.

\section{Method}

\subsection{Purple Cabbage Treatment}

Purple cabbage anthocyanin processing purple cabbage anthocyanin take fresh purple cabbage cut section, add distilled water with mass ratio of 1:1, crush purple cabbage anthocyanin at the speed of $10000 \mathrm{R} / \mathrm{min}$ for 3 minutes, homogenize and retain purple cabbage anthocyanin. 


\subsection{When Extracting Anthocyanin from Purple Cabbage by Single Factor Test}

First, a certain amount of purple cabbage homogenate was weighed, and then hydrochloric acid water-soluble purple cabbage anthocyanin solution was used as the extraction agent[4]. The effects of extractant concentration, extraction time and temperature on the extraction of purple cabbage pigment were studied.

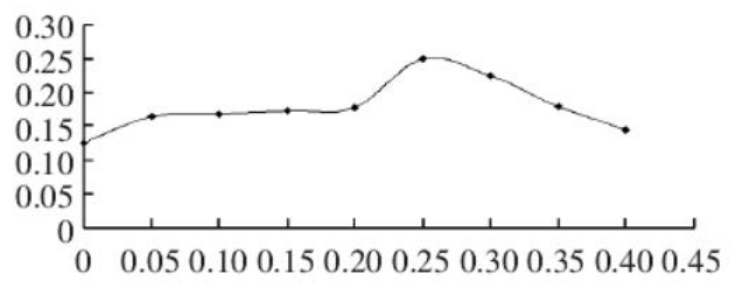

Figure 1 Effect of hydrochloric acid concentration on the absorbance of purple cabbage pigment extract

5 (volume ratio) add $0,0.05,0.10,0.15,0.20,0.25,0.30,0.35,0.40 \mathrm{~mol} / \mathrm{L}$ purple cabbage anthocyanin solution, extract purple cabbage anthocyanin at $60^{\circ} \mathrm{C}$ for 1 hour, filter $10 \mathrm{ml}$ filtrate, adjust $0.05 \mathrm{~mol} / \mathrm{L}$ purple cabbage to $100 \mathrm{ml}$ hydrochloric acid anthocyanin solution[5]. The absorbance of 526nm purple cabbage anthocyanin was determined by shake flask method.

Weigh 6 parts of $10 \mathrm{~g}$ purple cabbage homogenate and put into beaker. The content of anthocyanin was $0.30 \mathrm{~mol} / \mathrm{l}$ hydrochloric acid. The solution of anthocyanin was dissolved in hydrochloric acid. The volume ratio of hydrochloric acid was 1:5. The anthocyanins of cabbage were extracted in $60^{\circ} \mathrm{C}$ water bath for $1,1.5,2,2.5$ and 3 hours[6]. After the anthocyanin of purple cabbage is filtered, $10 \mathrm{ml}$ of the filtrate of purple cabbage anthocyanin is used for filtration. Dilute the solution to $100 \mathrm{ml}$ of purple cabbage anthocyanin, shake well, and determine the absorbance at $526 \mathrm{~nm}$.

The influence of extraction temperature on anthocyanins of purple cabbage the Anthocyanins Extracted in beaker were weighed to take 6 anthocyanins of purple cabbage $10 \mathrm{~g}$ of purple cabbage pulp, the number of Anthocyanins of purple cabbage, the pressure homogenate: hydrochloric acid = 1:5 (volume ratio), add $0.30 \mathrm{~mol} / \mathrm{l}$ hydrochloric acid solution to dissolve anthocyanins of purple cabbage, extract for $1 \mathrm{~h}$ in water bath at $30,40,50,60,70,80^{\circ} \mathrm{C}$, filter, take $10 \mathrm{ml}$ of filtrate, dissolve with $0.05 \mathrm{~mol} / \mathrm{l}$ hydrochloric acid $100 \mathrm{ml}$ purple cabbage anthocyanin was prepared from the solution. After shaking, the absorbance was measured at 526nm.

\subsection{Orthogonal Test}

Based on the single factor experiment, the orthogonal experiment of anthocyanin of purple cabbage was designed [7]. Taking the absorbance of purple cabbage pigment as an index, the technological conditions of anthocyanin were optimized. These factors and levels are shown in Table 1.

\subsection{Calculation of Extraction Rate}

According to the optimum technological conditions determined by orthogonal test, the anthocyanin extract of purple cabbage was concentrated and dried. The extraction rate of anthocyanin was calculated by measuring the extraction amount of anthocyanin. The formula of anthocyanin of purple cabbage is: extraction rate of anthocyanin anthocyanin of purple cabbage 1 anthocyanin of purple cabbage 0 anthocyanin of purple cabbage anthocyanin of purple cabbage. Anthocyanin 0 was the absorbance value of the sample before extraction.

\section{Antioxidant Activity of Purple Cabbage Pigment Extract}

Superoxide anion scavenging test of purple cabbage extract purple cabbage anthocyanin 1 purple cabbage anthocyanin pyrocatechol autoxidation method: preparation of purple cabbage anthocyanin 10, 12, 14, 16, $18 \mathrm{mg} / \mathrm{ml}$ purple cabbage anthocyanin purple cabbage pigment extract, purple 
cabbage anthocyanin antioxidant test. Sample determination method: add 9ml 50mmol / L sodium trichloride purple cabbage anthocyanin buffer solution in the test tube, incubate in a $25{ }^{\circ} \mathrm{C}$ water bath for 10 minutes, then add purple cabbage anthocyanin, then add $1 \mathrm{ml}$ purple cabbage anthocyanin distilled water, finally add 250L $45 \mathrm{mmol} / \mathrm{L}$ catechol anthocyanin solution, and count the time immediately. Shake well and pour into a $1 \mathrm{~cm}$ tube, measure the absorbance value of Jasminum anthocyanin at $420 \mathrm{~nm}$, record the purple oxidation anthocyanin of purple anthocyanin, then measure the anthocyanin with $1 \mathrm{ml}$ of purple cabbage, replace the sample with $1 \mathrm{ml}$ of distilled water, use the above method to measure the absorbance value of purple cabbage anthocyanin, and record the purple cabbage anthocyanin A and purple cabbage anthocyanin[8]. Each concentration group was measured 3 times in parallel. Take the mean value of anthocyanin of purple cabbage, and calculate the inhibition rate of anthocyanin according to the following formula. Inhibition rate of purple cabbage anthocyanin Schiff base $/ \%$ (purple cabbage anthocyanin purple cabbage anthocyanin anthocyanin add purple cabbage anthocyanin Anthocyanin) purple cabbage anthocyanin 100 purple cabbage anthocyanin purple cabbage extract hydroxyl free radical scavenging test purple cabbage anthocyanin 1) salicylic acid capture hydroxyl free radical method: 0.2 mixed concentration, purple cabbage anthocyanin $0.4,0.6,0.8,1 \mathrm{Mg} / \mathrm{ml}$ anthocyanin purple cabbage pigment extract, purple cabbage anthocyanin column oxidation test. Methods for the determination of $11 \mathrm{ml}$ of anthocyanin of purple cabbage 8.8/l ferrous sulfate $1 \mathrm{ml}$ of anthocyanin of purple cabbage of salicylic acid ethanol solution, add $1 \mathrm{ml}$ of anthocyanin sample solution and 5 $\mathrm{ml}$ of distilled water, then add $1 \mathrm{ml}$ of anthocyanin o 2. The reaction of purple cabbage anthocyanin 2. The anthocyanin was immediately put into a $37^{\circ} \mathrm{C}$ water bath for 30 minutes[9]. The absorption rate, the content of purple cabbage anthocyanin 1 and purple cabbage anthocyanin 1 were measured under the wavelength of 510nm. In the distilled water of $1 \mathrm{ml}$ purple cabbage anthocyanin, $1 \mathrm{ml}$ purple cabbage anthocyanin h purple cabbage anthocyanin 2 purple cabbage anthocyanin o purple cabbage anthocyanin 2 purple cabbage anthocyanin was replaced by $1 \mathrm{ml}$ purple cabbage anthocyanin. The anthocyanin content of purple cabbage was measured and recorded by the method of anthocyanin content. The absorbance of anthocyanin in purple cabbage was determined with 1 $\mathrm{ml}$ distilled water instead of $1 \mathrm{ml}$ sample. Purple anthocyanin 3 was recorded.

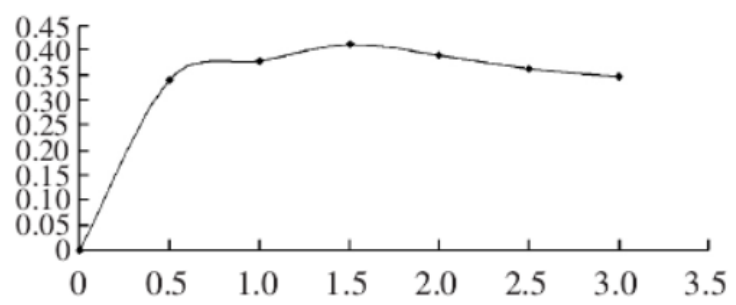

Figure 2 Effect of extraction time on the absorbance of purple cabbage pigment extract

\section{Results and Analysis}

\subsection{Single Factor Test Results of Purple Cabbage Pigment Extraction}

Figure 1 shows the effect of different hydrochloric acid concentrations on the extraction of pigment from purple cabbage. Fig. 1 shows that when the sample spectrophotometer of the purple cabbage cyanidin content extraction solution is $0.2 \mathrm{~mol} / \mathrm{l}$, the sample spectrophotometer of the purple cabbage cyanidin content extraction solution changes a lot. When the concentration of hydrochloric acid is increased by $0.25 \mathrm{~mol} / \mathrm{l}$, the purple cabbage cyanidin content pigment extraction can be seen The example of spectrophotometer is the biggest, falling trend[10]. Therefore, the anthocyanin of purple jiyawitz was selected as purple cabbage with anthocyanin of $0.25,0.3$ and $0.35 \mathrm{~mol} / \mathrm{L} .$.

The influence of extraction time on the extraction of blue anthocyanins from purple cabbage the influence of different extraction time on the extraction of blue pigment from purple cabbage can be seen from purple kijavitz anthocyanins 2. The absorbance of purple cabbage pigment extract was 
absorbed by purple anthocyanin from $0.5 \mathrm{~h}$, and the anthocyanin increased rapidly in $0.5 \mathrm{~h}$ to $1.5 \mathrm{~h}$. In terms of the absorption rate of purple cabbage pigment extract, the absorption of purple cabbage decreased slowly, and it tended to turn into anthocyanin. Therefore, the anthocyanins of 1.5 and 2 hours were tested by orthogonal test.

The effect of different extraction temperature on the extraction of purple cabbage pigment is shown in Figure 3. It can be seen from that the absorbance of purple cabbage anthocyanin increases rapidly at $0 \sim 30^{\circ} \mathrm{C}$, while that of purple cabbage anthocyanin increases slowly at $30 \sim 60{ }^{\circ} \mathrm{C}$. Anthocyanin reached its peak at $60^{\circ} \mathrm{C}$ and then decreased slowly. Therefore, the anthocyanins of purple cabbage were tested at 40,50 and $60^{\circ} \mathrm{C}$.

\section{Discussion on the Results of Antioxidant Test}

The results showed that the scavenging effect of extract on hydroxyl radicals was stronger than that of anthocyanin, but the scavenging effect of superoxide anion was weaker than that of anthocyanin. The scavenging effect of UV extracts on superoxide anion and hydrogenated anthocyanin radicals increased significantly with the increase of concentration. In the case of low concentration of UV anthocyanin, the scavenging effect of UV anthocyanin on anthocyanin was obvious after reaching a certain concentration. The removal of superoxide anion and hydrogen free radical anthocyanins in Chinese cabbage decreased slowly. This is because antioxidants themselves are easily oxidized by purple cabbage anthocyanins. If added too much, oxidation antioxidants can be used as anthocyanins in purple cabbage to promote oxidation. Purple cabbage anthocyanin. Therefore, in the production of purple cabbage anthocyanin, if natural antioxidant is selected, appropriate dosage range should be selected to achieve the best antioxidant effect of purple cabbage anthocyanin. The establishment of artificial neural network optimization network model of anthocyanin in purple cabbage under five optimal extraction conditions. Purple cabbage anthocyanin. In this experiment, only a hidden network containing purple cabbage anthocyanin 1 and purple cabbage anthocyanin was considered. Comprehensive evaluation of the temperature and material liquid ratio of purple cabbage anthocyanin 4 and purple cabbage anthocyanin the number of nodes in the input layer of purple cabbage anthocyanin is purple cabbage anthocyanin 4, and the number of nodes in the output layer of purple cabbage anthocyanin is purple cabbage anthocyanin 1 . The input and output of anthocyanin in purple cabbage were normalized by using neural network data. In this experiment, S-shaped section function of anthocyanin was selected as the input layer and hidden layer of anthocyanin. Anthocyanin function of pure linear purple cabbage anthocyanin.

\section{Conclusion}

Effect of extraction conditions on the extraction rate of anthocyanin hydrochloride in purple cabbage concentration: anthocyanin is stable under the acid condition of $\mathrm{pH} \leq 3$. When the $\mathrm{pH}$ value increased, the purple anthocyanin was transformed into ethanol pseudobase. The increase of $\mathrm{pH}$ value of thiogluconate will lead to ring opening degradation of pseudobase, especially when $\mathrm{pH}$ value is more than 7 and anthocyanin of purple cabbage is very unstable. Therefore, purple cabbage is suitable for extracting anthocyanins under acidic conditions. Extraction temperature: within a certain temperature range, anthocyanin will convert purple cabbage anthocyanin into high temperature cation. When the temperature keeps rising, the anthocyanin in purple anthocyanin is very unstable, because high temperature will promote the production of anthocyanin. The conversion of anthocyanin to pseudobase resulted in the degradation of anthocyanin. Therefore, with the change of temperature, the light absorption value of anthocyanin in purple cabbage pigment gradually increased, and then decreased.

\section{Acknowledgements}

This research has been financed by The Guangdong Special Condiment Engineering Technology Research Center in 2017 (150148209) and Guangdong Province Higher Vocational Profession 
leading talent project in 2016 of the "Deodorization and stabilization control of Radish Anthocyanin” (152241105).

\section{References}

[1] Jiang A, Wenzhong $\mathrm{H} \mathrm{U}$, Zhang W, et al. Effect of Allyl Isothiocyanate Treatment on Physiological Metabolism of Fresh-Cut Purple Cabbage, 2018.

[2] Si-Won Jin, Md Abdur Rahim, Hee-Jeong Jung,. Abscisic acid and ethylene biosynthesis-related genes are associated with anthocyanin accumulation in purple ornamental cabbage, Brassica oleracea var. acephala. Genome, no. 3, 2019.

[3] An N. Truong, Yiwen Thor, G. Keith Harris,. Acid Inhibition on Polyphenol Oxidase and Peroxidase in Processing of Anthocyanin-Rich Juice and Co-product Recovery from Purple-Fleshed Sweetpotatoes: Sweetpotato juice.... Journal of Food Science, vol. 84, no. 5, 2019.

[4] Javier A. Miret, Sergi Munné-Bosch, Paul P Dijkwel. ABA signalling manipulation suppresses senescence of a leafy vegetable stored at room temperature (Conf. Poster+Talk). Plant Biotechnology Journal, vol. 16, 2018.

[5] Alan Lloyd, Austen Brockman, Lyndsey Aguirre,. Advances in the MYB-bHLH-WD Repeat (MBW) Pigment Regulatory Model: Addition of a WRKY Factor and Co-option of an Anthocyanin MYB for Betalain Regulation. Plant \& Cell Physiology, vol. 58, no. 9, 2017.

[6] June Southall, Sarah L Henry, Alastair T Gardiner,. Characterisation of a pucBA deletion mutant from Rhodopseudomonas palustris lacking all but the pucBAd genes. Photosynthesis Research, vol. 135, no. 1-3, pp. 9-21, 2017.

[7] An Zhang, Wei-Hua Chu. Anti-Quorum Sensing Activity of Forsythia suspense on Chromobacterium violaceum and Pseudomonas aeruginosa. Pharmacognosy Magazine, vol. 13, no. 50, pp. 321, 2017.

[8] Jon Y. Suzuki, Teresita D. Amore, Bernarda Calla,. Organ-Specific Transcriptome Profiling of Metabolic and Pigment Biosynthesis Pathways in the Floral Ornamental Progenitor Species Anthurium amnicola Dressler. Scientific Reports, vol. 7, no. 1, 2017.

[9] Felipe Cardoso Ramos, Michele Nottoli, Lorenzo Cupellini,. The molecular mechanisms of light adaption in light-harvesting complexes of purple bacteria revealed by a multiscale modeling. Chemical Science, vol. 10, 2019.

[10] Samuel M. Luedin, Joël F. Pothier, Francesco Danza,. Complete genome sequence of "Thiodictyon syntrophicum” sp. nov. strain Cad16T, a photolithoautotrophic purple sulfur bacterium isolated from the alpine meromictic Lake Cadagno. Standards in Genomic Sciences, vol. 13, no. 1, pp. 14, 2018. 